\title{
Nonlinear Oscillations of CNT Nano-resonator Based on Nonlocal Elasticity: The Energy Balance Method
}

\author{
Masoud Goharimanesh ${ }^{\mathbf{1}}$ and Ali Koochi ${ }^{\mathbf{2}}$ \\ ${ }^{1}$ Department of Mechanical Engineering, University of Torbat Heydarieh, Torbat Heydarieh, Iran, Email: \\ M.goharimanesh@torbath.ac.ir \\ ${ }^{2}$ Department of Mechanical Engineering, University of Torbat Heydarieh, Torbat Heydarieh, Iran, Email:
}

A.koochi@torbath.ac.ir

\begin{tabular}{l} 
Article Info \\
\hline Article history: \\
Received November 28, 2020 \\
Revised February 15, 2021 \\
Accepted February 18, 2021 \\
\hline
\end{tabular}

\section{Keywords:}

Carbo nanotube,

Casimir force,

Energy balance method,

Nano-resonator,

Nonlocal elasticity.

\begin{abstract}
This paper deals with investigating the nonlinear oscillation of carbon nanotube manufactured nano-resonator. The governing equation of the nanoresonator is extracted in the context of the nonlocal elasticity. The impact of the Casimir force is also incorporated in the developed model. A closed-form solution based on the energy balance method is presented for investigating the oscillations of the nano-resonator. The proposed closed-form solution is compared with the numerical solution. The impact of influential parameters including applied voltage, Casimir force, geometrical and nonlocal parameters on the nano resonator's vibration and frequency are investigated. The obtained results demonstrated that the Casimir force reduces the nano-resonator frequency. However, the nonlocal parameter has a hardening effect and enhances the system's frequency.
\end{abstract}

Copyright (C) 2020 Regional Association for Security and crisis management and European centre for operational research. All rights reserved.

\section{Corresponding Author:}

Ali Koochi,

Department of Mechanical Engineering, University of Torbat Heydarieh, Torbat Heydarieh, Iran.

Email: A.koochi@torbath.ac.ir

\section{Introduction}

Owing to their remarkable electrical and mechanical properties, carbon nanotubes (CNTs) have become of great interest to a wide range of applications in science and technology. CNTs have potential for broad application in ultra-small devices including, but not limited to, sensor (Ali-Akbari et al., 2019; Kang et al., 2017), resonators (Besley, 2020; Ouakad \& Younis, 2010), tweezers (Farrokhabadi et al., 2014; Kim \& Lieber, 1999), probes (Y. Liu et al., 2017), actuators(Sedighi \& Farjam, 2017) and switches (Dequesnes et al., 2004). With the new demands for ultra-small high accuracy devices, miniature resonators become the building block of modern instruments such as sensors (Hajjam \& Pourkamali, 2011; Qian et al., 2016), accelerometers (Tocchio et al., 2011), and communication and signal processing devices (Mestrom et al., 2008).

Simulating and investigating micro/nano resonators' dynamic performances are essential steps prior to manufacturing these systems. Hence, previous researchers have focused on exploring the phenomena affecting the behavior of micro/nano-resonators. A simple one degree of freedom model for investigating the chaotic regime of electromechanical nano-resonator was presented by Amorim et al. (2015). They considered the impacts of the Casimir force in their simulation. While using the one degree of freedom model can considerably simplify the simulating and analyzing of the nano-resonator, its result might be unacceptable. To overcome this, some researchers use the continuum models for investigating the dynamic performances of these devices. Miandoab et al. (2015) analyzed the dynamic and chaos in an electromechanically nano-resonator. They include the impact of both DC and AC voltage in their simulation. The influence of the material size dependency on the dynamic performance of nano-resonator investigated by Miandoab et al. (2014). To this 
end, they developed the governing equation of nano-resonator based on the strain gradient elasticity. A homotopy analysis method is employed for investigating the nonlinear dynamic of double side resonator by Tajaddodianfar et al. (2017). Dantas and Gusso (2018) investigated the chaotic dynamics of a MEMS resonator. They simulated the resonator based on the classical Euler-Bernoulli beam model.

Within the classical continuum theories, the stresses at each point are just related to stains at the same point. Nevertheless, the stresses at one point of the nonlocal elasticity are related to the strains at the whole body. This assumption incorporates the internal length-scale as an extra material factor in the constitutive equations. The nonlocal elasticity was introduced by Eringen and Edelen (1972). This theory has been employed for investigating the impact of scale dependency of CNT based structures (Khosravi et al., 2020; H. Liu et al., 2019; Pashaki \& Ji, 2020; Rahmani et al., 2018; Yang et al., 2018; Zeighampour et al., 2017). Hosseini (2018) simulated the heat effected nano-beam resonator based on the nonlocal thermoelectricity. He considered the thermal shock loading as an actuating method instead of electrostatic actuation

In this research article, a closed-form solution based on the energy balance method is presented for nonlocal dynamic analysis of CNT based nano-resonator. The nano-resonator is simulated as a double clamped CNT between two fixed plates. The CNT deformation is modeled in the context of nonlocal Euler Bernoulli beam theory. Then, with the help of Hamilton's principle, the nano-resonator's governing equation is developed. Also, the impact of the Casimir force is taken into account in the developed model. The partial differential equation of the system is transformed into a nonlinear ordinary differential equation using the Galerkin procedure. Then, the energy balance method is employed, and a close form solution for oscillations of nanoresonator is established. To examine the accuracy of the solution, he proposed a closed-form solution is compared to a numerical simulation. Finally, the impact of small-scale parameters, Casimir force, CNT geometry on the amplitude and frequency of the vibrations are examined.

\section{Theoretical Model}

Figure 1 shows a CNT based nano-resonator. The nano-resonator is constructed from a clamped-clamped CNT fixed between two plates. The mean radius of the CNT is $R_{w}$, and its length is $L$. Also, the gap between the CNT and both the upper and the lower plate is $g$.

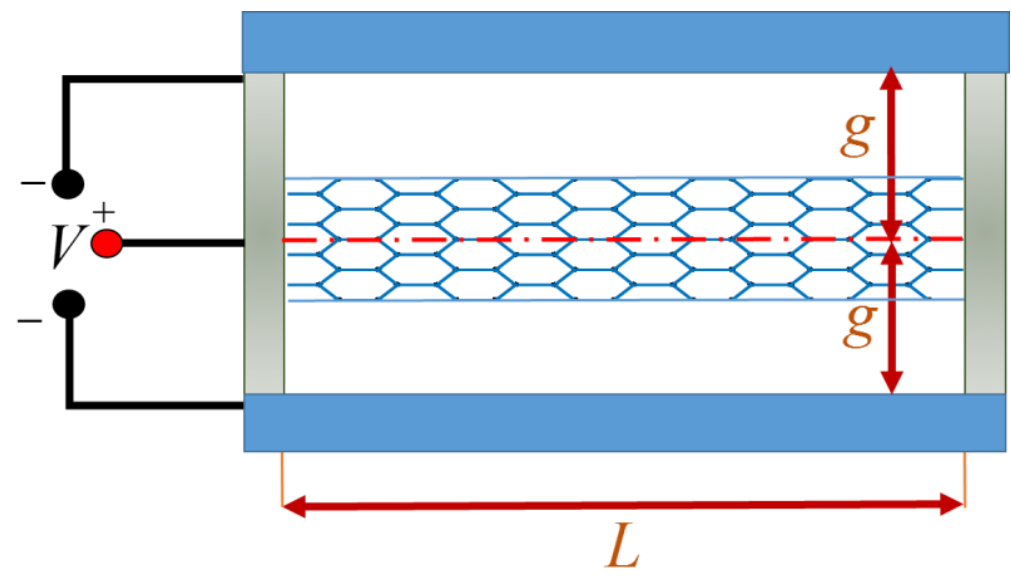

Figure 1. A CNT based nano-resonator

We model the nano resonator as a clamped-clamped Euler-Bernoulli beam. Therefore, the CNT deformation can be explained as:

$$
u_{1}=\hat{u}(\hat{x}, t)-\hat{z} \frac{\partial \hat{w}(\hat{x}, t)}{\partial \hat{x}}, \quad u_{2}=0, \quad u_{3}=\hat{w}(\hat{x}, t)
$$

The stain energy of the CNT is given as:

$$
U=\frac{1}{2} \int_{0}^{L} \int_{A} \boldsymbol{\sigma} . \varepsilon \cdot d A d \hat{x}
$$

where $U$ is the strain energy, $\sigma$ indicated the stress tensor and $\varepsilon$ is the strain tensor. Using the displacement field of Eq. (1), the non-zero component of strain tensor is obtained as: 


$$
\varepsilon_{\hat{x} \hat{x}}=\frac{\partial \hat{u}}{\partial \hat{x}}-\hat{z} \frac{\partial^{2} \hat{w}}{\partial \hat{x}^{2}}+\frac{1}{2}\left(\frac{\partial \hat{w}}{\partial \hat{x}}\right)^{2}
$$

Substituting Eq. (3) in Eq. (1), the strain energy of the nano-resonator is defined as:

$$
\left.U=\frac{1}{2} \int_{0}^{L} \int_{A} \sigma_{\hat{x} \hat{x}} \varepsilon_{\hat{x}} d A d \hat{x}=\frac{1}{2} \int_{0}^{L}\left(-M^{n l} \frac{\partial^{2} \hat{w}}{\partial \hat{x}^{2}}+N^{n l}\left(\frac{\partial \hat{u}}{\partial \hat{x}}+\frac{1}{2}\left(\frac{\partial \hat{w}}{\partial \hat{x}}\right)^{2}\right)\right)\right) d \hat{x}
$$

The work of the external loads $(F(\hat{x}, t))$, is explained as:

$$
W=\int_{0}^{L} \int_{0}^{\hat{w}} F(\hat{x}, t) \mathrm{d} \hat{w} \mathrm{~d} \hat{x}
$$

It is worth to note that the external load exerting on the nano-resonator is the summation of the electrostatic and Casimir forces.

The electrical force acts on the nano-tweezers can be determined by using the capacitor model. We separate the electrical force to the upper and lower plate contributions. The electrical force $\left(\mathrm{F}_{\text {elc }}\right)$ between the CNT and a plate can be explained as (Koochi et al., 2015):

$$
F_{\text {elec }}=\frac{\pi \varepsilon_{0} V^{2}}{g \ln ^{2}\left(2 \frac{g}{R_{w}}\right)}
$$

In the above equation $\varepsilon_{0}$ is the vacuum permittivity and $V$ is the applied voltage. When the nano-resonator oscillates, the distance between the upper plate and CNT becomes $g+\hat{w}(t)$, and the distance between the upper plate and CNT becomes $g-\hat{w}(t)$. Therefore, the upper and lower electrical load for vibrating nano-resonator are defined as:

$$
\begin{gathered}
F_{\text {elec }}^{\text {upper }}=-\frac{\pi \varepsilon_{0} V^{2}}{(g+\hat{w}) \ln ^{2}\left(2 \frac{g+\hat{w}}{R_{w}}\right)} \\
F_{\text {elec }}^{\text {lower }}=\frac{\pi \varepsilon_{0} V^{2}}{(g-\hat{w}) \ln ^{2}\left(2 \frac{g-\hat{w}}{R_{w}}\right)}
\end{gathered}
$$

Where the superscript upper and lower indicate the force due to upper and lower plate, respectively. Similar to electrical force, the Casimir attraction is due to the upper and lower plates. For a cylinder over a plate, the Casimir force $\left(\mathrm{F}_{\mathrm{Cas}}\right)$ is given as:

$$
F_{\text {Cas }}=\frac{\hbar c}{8 g^{3} \pi \ln \left(\frac{g}{R_{W}}\right)}+\frac{\hbar c}{16 g^{3} \pi \ln ^{2}\left(\frac{g}{R_{W}}\right)} \text {. }
$$

where $\hbar$ is the reduced Planck's constant, and $c$ is the speed of light. For vibrating nano-resonator, the contributions of the upper and lower plate on the Casimir force are defined as:

$$
\begin{aligned}
& F_{\text {Cas }}^{\text {upper }}=-\frac{\hbar c}{16(g+\hat{w})^{3} \pi \ln ^{2}\left(\frac{g+\hat{w}}{R_{W}}\right)}\left[1+2 \ln \left(\frac{g+\hat{w}}{R_{W}}\right)\right] \\
& F_{\text {Cas }}^{\text {lower }}=\frac{\hbar c}{16(g-\hat{w})^{3} \pi \ln ^{2}\left(\frac{g-\hat{w}}{R_{W}}\right)}\left[1+2 \ln \left(\frac{g-\hat{w}}{R_{W}}\right)\right]
\end{aligned}
$$

The beam's kinetic energy (T) is described as:

$$
\begin{aligned}
& T=\frac{1}{2} \int_{o}^{L} \int_{A} \rho\left[\left(\frac{\partial \hat{u}}{\partial t}-Z \frac{\partial^{2} \hat{w}}{\partial t \partial \hat{x}}\right)^{2}+\left(\frac{\partial \hat{w}}{\partial t}\right)^{2}\right] d A d \hat{x} \\
& =\frac{1}{2} \int_{0}^{L}\left[\rho A\left(\frac{\partial \hat{u}}{\partial t}\right)^{2}+\rho A(\hat{w})^{2}+\rho I\left(\frac{\partial^{2} \hat{w}}{\partial t \partial \hat{x}}\right)^{2}\right] d \hat{x}
\end{aligned}
$$

where $\rho$ is the mass density. Hamilton's principle is employed to extract the CNT based nano-sensor governing equation: 
$\int_{t_{1}}^{t_{2}}(\delta T-\delta U+\delta W) d t=0$

Replacing Eqs. (4), (5) and (12) in Eq. (13) and after some mathematical elaborations, one obtains:

$$
\begin{aligned}
& \frac{\partial N^{n l}}{\partial \hat{x}}=\rho A \frac{\partial^{2} \hat{u}}{\partial t^{2}} \\
& \rho A \frac{\partial^{2} \hat{w}}{\partial t^{2}}+\frac{\partial}{\partial \hat{x}}\left(N \frac{\partial \hat{w}}{\partial \hat{x}}\right)-\rho I \frac{\partial^{4} \hat{w}}{\partial t^{2} \partial \hat{x}^{2}}-\frac{\partial^{2} M^{n l}}{\partial \hat{x}^{2}}=F(\hat{x}, t)
\end{aligned}
$$

with the boundary conditions below:

$$
\begin{aligned}
& \hat{w}(0)=\hat{w}(L)=0 \\
& \frac{\partial \hat{w}}{\partial \hat{x}}(0)=\frac{\partial \hat{w}}{\partial \hat{x}}(L)=0 \\
& \hat{u}(0)=\hat{u}(L)=0
\end{aligned}
$$

The nonlocal constitutive equation for bending moment and normal force can be explained as (Mahmoud et al., 2012):

$$
\begin{aligned}
& \left(1-e_{0}^{2} a^{2} \frac{\partial^{2}}{\partial \hat{x}^{2}}\right) M^{n l}=M^{l} \\
& \left(1-e_{0}^{2} a^{2} \frac{\partial^{2}}{\partial \hat{x}^{2}}\right) N^{n l}=N^{l}
\end{aligned}
$$

where the superscript $l$ indicates the local force and moments and superscript $n l$ indicates the nonlocal force and moment. Also, $a$ is the internal characteristic length, and $e_{0}$ is a constant appropriate to each material. The local moment of bending $\left(M^{l}\right)$ and the normal force $\left(N^{l}\right)$ are described as:

$$
\begin{aligned}
& M^{l}=\int_{A} \hat{z} \sigma_{\hat{x}}^{l} d A=-E I \frac{\partial^{2} \hat{w}}{\partial \hat{x}^{2}} \\
& N^{l}=\int_{A} \sigma_{\hat{x}}^{l} d A=E A\left(\frac{\partial \hat{u}}{\partial \hat{x}}+\frac{1}{2}\left(\frac{\partial \hat{w}}{\partial \hat{x}}\right)^{2}\right)
\end{aligned}
$$

Replacing Eqs. (14) and (15) in Eqs. (17) and (18) results in:

$$
\begin{aligned}
& N^{n l}=e_{0}^{2} a^{2} \rho A \frac{\partial^{3} \hat{u}}{\partial \hat{x} \partial t^{2}}+E A\left(\frac{\partial \hat{u}}{\partial \hat{x}}+\frac{1}{2}\left(\frac{\partial \hat{w}}{\partial \hat{x}}\right)^{2}\right) \\
& M^{n l}=-E I \frac{\partial^{2} \hat{w}}{\partial \hat{x}^{2}}+e_{0}^{2} a^{2}\left(\rho A \frac{\partial^{2} \hat{w}}{\partial t^{2}}-\rho I \frac{\partial^{4} W}{\partial t^{2} \partial \hat{x}^{2}}+\frac{\partial}{\partial X}\left(N \frac{\partial \hat{w}}{\partial \hat{x}}\right)-F(\hat{x}, t)\right)
\end{aligned}
$$

Replacing Eqs. (15) and (16) in Eq. (13), the nonlocal constitutive equation are obtained as:

$$
\begin{aligned}
& \left(1-e_{0}^{2} a^{2} \frac{\partial^{2}}{\partial \hat{x}^{2}}\right) \rho A \frac{\partial^{2} \hat{u}}{\partial t^{2}}=\frac{\partial}{\partial \hat{x}}\left[E A\left(\frac{\partial \hat{u}}{\partial \hat{x}}+\frac{1}{2}\left(\frac{\partial \hat{w}}{\partial \hat{x}}\right)^{2}\right)\right] \\
& \left(1-e_{0}^{2} a^{2} \frac{\partial^{2}}{\partial \hat{x}^{2}}\right)\left[\rho A \frac{\partial^{2} \hat{w}}{\partial t^{2}}-\rho I \frac{\partial^{4} \hat{w}}{\partial t^{2} \partial \hat{x}^{2}}\right]+E I \frac{\partial^{4} \hat{w}}{\partial \hat{x}^{4}} \\
& +\frac{\partial}{\partial \hat{x}}\left(E A\left(\frac{\partial \hat{u}}{\partial \hat{x}}+\frac{1}{2}\left(\frac{\partial \hat{w}}{\partial \hat{x}}\right)^{2}\right) \frac{\partial \hat{w}}{\partial \hat{x}}\right)=\left(1-e_{0}^{2} a^{2} \frac{\partial^{2}}{\partial \hat{x}^{2}}\right) F(\hat{x}, t)
\end{aligned}
$$

Where $A$ is the cross-section area and $I$ is the cross-section second moment of area. By neglecting the longitudinal inertia, Eq. (17) can be replaced with the following equation:

$$
\begin{aligned}
& \rho A\left(1-e_{0}^{2} a^{2} \frac{\partial^{2}}{\partial \hat{x}^{2}}\right) \frac{\partial^{2} \hat{w}}{\partial t^{2}}+E I \frac{\partial^{4} \hat{w}}{\partial \hat{x}^{4}}-\left[\frac{E A}{2 L} \int_{0}^{L}\left(\frac{\partial \hat{w}}{\partial \hat{x}}\right)^{2} d X\right] \frac{\partial^{2} \hat{w}}{\partial \hat{x}^{2}} \\
& =\left(1-e_{0}^{2} a^{2} \frac{\partial^{2}}{\partial \hat{x}^{2}}\right) F(\hat{x}, t)
\end{aligned}
$$


Substituting the electrical and Casimir force in the above equation, the nonlocal governing equation of CNT based nano-resonator is achieved as:

$$
\begin{aligned}
& \rho A\left(1-e_{0}^{2} a^{2} \frac{\partial^{2}}{\partial \hat{x}^{2}}\right) \frac{\partial^{2} \hat{w}}{\partial t^{2}}+E I \frac{\partial^{4} \hat{w}}{\partial \hat{x}^{4}}-\left[\frac{E A}{2 L} \int_{0}^{L}\left(\frac{\partial \hat{w}}{\partial \hat{x}}\right)^{2} d X\right] \frac{\partial^{2} \hat{w}}{\partial \hat{x}^{2}} \\
& =\left(1-e_{0}^{2} a^{2} \frac{\partial^{2}}{\partial \hat{x}^{2}}\right)\left[\frac{\pi \varepsilon_{0} V^{2}}{(g-\hat{w}) \ln ^{2}\left(2 \frac{g-\hat{w}}{R_{w}}\right)}-\frac{\pi \varepsilon_{0} V^{2}}{\left.(g+\hat{w}) \ln ^{2}\left(2 \frac{g+\hat{w}}{R_{w}}\right)\right]}\right. \\
& +\frac{\hbar c\left(1-e_{0}^{2} a^{2} \frac{\partial^{2}}{\partial \hat{x}^{2}}\right)}{16 \pi}\left[\frac{\left[1+2 \ln \left(\frac{g-\hat{w}}{R_{W}}\right)\right]}{(g-\hat{w})^{3} \ln ^{2}\left(\frac{g-\hat{w}}{R_{w}}\right)}-\frac{\left[1+2 \ln \left(\frac{g+\hat{w}}{R_{W}}\right)\right]}{(g+\hat{w})^{3} \ln ^{2}\left(\frac{g+\hat{w}}{R_{w}}\right)}\right]
\end{aligned}
$$

The dimensionless motion equation could be reached by considering the following parameters:

$$
\begin{aligned}
& \alpha=\left(\frac{e_{0} a}{L}\right)^{2} ; k=\frac{A g^{2}}{2 I} ; \gamma=\frac{g}{R_{w}} ; x=\frac{\hat{x}}{L} ; \\
& w=\frac{\hat{w}}{g} ; \tau=\sqrt{\frac{E I}{\rho A L^{4}}} t ;=\frac{V L^{2}}{g} \sqrt{\frac{\pi \varepsilon_{0} \varepsilon_{r}}{E I}} ; \lambda=\frac{\hbar c L^{4}}{16 g^{4} E I}
\end{aligned}
$$

Using Eq. (39), we have:

$$
\begin{aligned}
& \left(1-\alpha \frac{\partial^{2}}{\partial x^{2}}\right) \frac{\partial^{2} w}{\partial \tau^{2}}+\frac{\partial^{4} w}{\partial x^{4}}-\left[k \int_{0}^{1}\left(\frac{\partial w}{\partial x}\right)^{2} d x\right] \frac{\partial^{2} w}{\partial x^{2}}= \\
& \left(1-\alpha \frac{\partial^{2}}{\partial x^{2}}\right)\left[\frac{\eta^{2}}{(1-w) \ln ^{2}(2 \gamma(1-w))}-\frac{\eta^{2}}{(1+w) \ln ^{2}(2 \gamma(1+w))}\right] \\
& \left(1-\alpha \frac{\partial^{2}}{\partial x^{2}}\right)\left[\frac{\lambda[1+2 \ln (\gamma(1-w))]}{(1-w)^{3} \ln ^{2}(\gamma(1-w))}-\frac{\lambda[1+2 \ln (\gamma(1+w))]}{(1+w)^{3} \ln ^{2}(\gamma(1+w))}\right]
\end{aligned}
$$

\section{Solution}

The idea of the energy balance method comes from the fact that for an oscillation system with the frequency of $\omega$, when $\omega t=n \pi$, the whole energy of the system is in the form of the kinetic energy. Similarly, when $\omega t=\frac{2 n+1}{2} \pi$, the velocity is zero and the whole energy of the system is in the form of the potential energy $(n=0,1,2, .$.$) . Hence, in \omega t=\pi / 4$, a balance between the kinetic and the potential energy of the system can be assumed. The energy balance method utilizes the advantage of this point to collocate a solution at $\omega t=\pi / 4$. While the idea of the energy balance method is straightforward, the results are valid for investigating the vibration of some highly nonlinear systems. Interestingly, even the lowest order approximations are of high accuracy (Fu et al., 2011; Ganji et al., 2009; Ghalambaz et al., 2015; Jamshidi \& Ganji, 2010; Mehdipour et al., 2010).

To solve the nonlinear differential equation of the system based on the energy balance method, the displacement is separated into time-dependent and time-independent parts as:

$$
w(x)=q(\tau) \phi(x)
$$

We consider the first mode shape of the clamped-clamped beam as the time-independent part:

$$
\phi(x)=\cosh (\xi x)-\cos (\xi x)-\frac{\cosh (\xi)-\cos (\xi)}{\sinh (\xi)-\sin (\xi)}(\sinh (\xi x)-\sin (\xi x))
$$

where $\xi=4.73004074$. Multiplying both sides of Eq. (27) by $\phi(x)$ and integrating the result relation from 0 to 1 yields

$$
\frac{d^{2} q}{d \tau^{2}}+\Lambda_{0}+\Lambda_{1} q+\Lambda_{2} q^{2}+\Lambda_{3} q^{3}+\Lambda_{4} q^{4}+\Lambda_{5} q^{5}+\Lambda_{6} q^{6}=0
$$

where: 


$$
\begin{aligned}
& \Lambda_{0}=-\frac{0.8308610}{1+12.302618 \alpha} \\
& \Lambda_{1}=\frac{-12.302618 \mathrm{~F}_{1} \alpha-\mathrm{F}_{1}+\xi^{4}}{1+12.302618 \alpha} \\
& \Lambda_{2}=\frac{-19.140541 \mathrm{~F}_{2} \alpha-1.329378 \mathrm{~F}_{2}}{1+12.302618 \alpha} \\
& \Lambda_{3}=\frac{-28.213206 \mathrm{~F}_{3} \alpha-1.851896 \mathrm{~F}_{3}+151.354424 \mathrm{k}}{1+12.302618 \alpha} \\
& \Lambda_{4}=\frac{-41.572215 \mathrm{~F}_{4} \alpha-2.651096 \mathrm{~F}_{4}}{1+12.302618 \alpha} \\
& \Lambda_{5}=\frac{-61.654775 \mathrm{~F}_{5} \alpha-3.863603 \mathrm{~F}_{5}}{1+12.302618 \alpha} \\
& \Lambda_{6}=\frac{-92.066223 \mathrm{~F}_{6} \alpha-5.702139 \mathrm{~F}_{6}}{1+12.302618 \alpha}
\end{aligned}
$$

In Eq. (31):

$$
\begin{aligned}
& F_{m}=\frac{1}{m !} \frac{\partial}{\partial w^{m}}\left[\frac{\eta^{2}}{(1-w) \ln ^{2}(2 \gamma(1-w))}-\frac{\eta^{2}}{(1+w) \ln ^{2}(2 \gamma(1+w))}\right]_{w=0} \\
& +\frac{1}{m !} \frac{\partial}{\partial w^{m}}\left[\frac{\lambda[1+2 \ln (\gamma(1-w))]}{(1-w)^{3} \ln ^{2}(\gamma(1-w))}-\frac{\lambda[1+2 \ln (\gamma(1+w))]}{(1+w)^{3} \ln ^{2}(\gamma(1+w))}\right]_{w=0}
\end{aligned}
$$

A trial solution with the amplitude $\mathrm{A}$ and frequency $\omega$ is assumed as:

$$
w(\tau)=A \cos (\omega \tau)
$$

The initial conditions for Eq. (28) are considered as zero velocity and displacement magnitude A:

$$
\begin{aligned}
& q(0)=A \\
& \dot{q}(0)=0
\end{aligned}
$$

Following the energy balance method, the variational form of Eq. (30) is defined as:

$$
J(q)=\int_{o}^{\tau}\left(-\frac{1}{2} \dot{q}^{2}+\Lambda_{0} q+\frac{1}{2} \Lambda_{1} q^{2}+\frac{1}{3} \Lambda_{2} q^{3}+\frac{1}{4} \Lambda_{3} q^{4}+\frac{1}{5} \Lambda_{4} q^{5}+\frac{1}{6} \Lambda_{5} q^{6}+\frac{1}{7} \Lambda_{6} q^{7}\right) d \tau
$$

The Hamiltonian can be rewritten as:

$$
\begin{aligned}
& H=\frac{1}{2} \dot{q}^{2} \\
& +\Lambda_{0} q+\frac{1}{2} \Lambda_{1} q^{2}+\frac{1}{3} \Lambda_{2} q^{3}+\frac{1}{4} \Lambda_{3} q^{4}+\frac{1}{5} \Lambda_{4} q^{5}+\frac{1}{6} \Lambda_{5} q^{6}+\frac{1}{7} \Lambda_{6} q^{7}= \\
& +\Lambda_{0} A+\frac{1}{2} \Lambda_{1} A^{2}+\frac{1}{3} \Lambda_{2} A^{3}+\frac{1}{4} \Lambda_{3} A^{4}+\frac{1}{5} \Lambda_{4} A^{5}+\frac{1}{6} \Lambda_{5} A^{6}+\frac{1}{7} \Lambda_{6} A^{7}
\end{aligned}
$$

Substituting the trial solution in Eq. (36) results in:

$$
\begin{aligned}
& H=\frac{1}{2}(-A \omega \sin (\omega \tau))^{2}+\Lambda_{0}(A \cos (\omega \tau)-A)+\frac{1}{2} \Lambda_{1}\left(A^{2} \cos ^{2}(\omega \tau)-A^{2}\right) \\
& +\frac{1}{3} \Lambda_{2}\left(A^{3} \cos ^{3}(\omega \tau)-A^{3}\right)+\frac{1}{4} \Lambda_{3}\left(A^{4} \cos ^{4}(\omega \tau)-A^{4}\right)+\frac{1}{5} \Lambda_{4}\left(A^{5} \cos ^{5}(\omega \tau)-A^{5}\right) \\
& +\frac{1}{6} \Lambda_{5}\left(A^{6} \cos ^{6}(\omega \tau)-A^{6}\right)+\frac{1}{7} \Lambda_{6}\left(A^{7} \cos ^{7}(\omega \tau)-A^{7}\right)=0
\end{aligned}
$$

Solving Eq. (37) for $\omega$ results in:

$$
\omega=\frac{\sqrt{\left(\begin{array}{l}
\left.2 \Lambda_{0}(A-A \cos (\omega \tau))+\Lambda_{1}\left(A^{2}-A^{2} \cos ^{2}(\omega \tau)\right)+\frac{2}{3} \Lambda_{2}\left(A^{3}-A^{3} \cos ^{3}(\omega \tau)\right)\right) \\
+\frac{1}{2} \Lambda_{3}\left(A^{4}-A^{4} \cos ^{4}(\omega \tau)\right)+\frac{2}{5} \Lambda_{4}\left(A^{5}-A^{5} \cos ^{5}(\omega \tau)\right) \\
+\frac{1}{3} \Lambda_{5}\left(A^{6}-A^{6} \cos ^{6}(\omega \tau)\right)+\frac{2}{7} \Lambda_{6}\left(A^{7}-A^{7} \cos ^{7}(\omega \tau)\right)
\end{array}\right)}}{A \sin (\omega \tau)}
$$


Collocation Eq. (38) at $\pi / 4$ :

$$
\omega=\sqrt{\frac{\left(\begin{array}{l}
840 \Lambda_{0}(2-\sqrt{2})+105 \Lambda_{1} A+140 \Lambda_{2} A^{2}(4-\sqrt{2}) \\
+630 \Lambda_{3} A^{3}+84 \Lambda_{4} A^{4}(8-\sqrt{2}) \\
+35 \Lambda_{5} A^{5}+15 \Lambda_{6} A^{6}(6-\sqrt{2})
\end{array}\right)}{420 A}}
$$

Substituting Eq. (39) in Eq. (33) one obtains:

$$
q(\tau)=A \cos \left[\sqrt{\left.\frac{\left(\begin{array}{l}
840 \Lambda_{0}(2-\sqrt{2})+105 \Lambda_{1} A+140 \Lambda_{2} A^{2}(4-\sqrt{2}) \\
+630 \Lambda_{3} A^{3}+84 \Lambda_{4} A^{4}(8-\sqrt{2}) \\
+35 \Lambda_{5} A^{5}+15 \Lambda_{6} A^{6}(6-\sqrt{2})
\end{array}\right.}{420 A} \tau\right]}\right.
$$

Finally, the nonlinear oscillation of nano-resonator can be expressed as the following relation:

$$
w(x, \tau)=A \phi(x) \cos \left[\sqrt{\left.\frac{\left(\begin{array}{l}
840 \Lambda_{0}(2-\sqrt{2})+105 \Lambda_{1} A+140 \Lambda_{2} A^{2}(4-\sqrt{2}) \\
+630 \Lambda_{3} A^{3}+84 \Lambda_{4} A^{4}(8-\sqrt{2}) \\
+35 \Lambda_{5} A^{5}+15 \Lambda_{6} A^{6}(6-\sqrt{2})
\end{array}\right)}{420 A} \tau\right]}\right.
$$

\section{Results and discussion}

To investigate the accuracy of the proposed closed form solution, the nano-resonator amplitude as a function of dimensionless time parameter $(\tau)$ is presented in Figure 2. In this figure, the nonlocal dimensionless parameter is 0.4 , the Casimir force parameter is 1 , the electrical parameter is 2 , and the geometrical parameter is 20 . Figure 2 demonstrates that the results of the energy balance method are entirely close to the numerical solution.

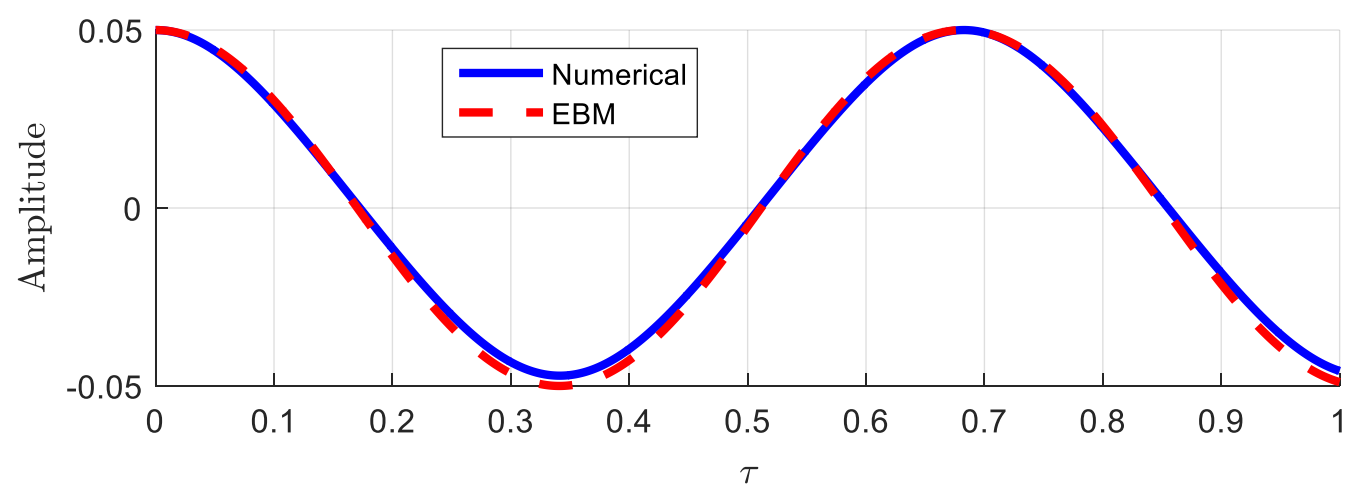

Figure 2. Comparison between the energy balance method and the numerical solution

Figure 3 shows the impact of the geometrical parameter and the applied voltage on the nano-resonator's frequency. In this figure, the nonlocal dimensionless parameter is 0.8 , the Casimir force parameter is 2 , and the geometrical parameter is 20 . Figure 3 demonstrates that the frequency of the nano-resonator enhances by increasing the geometrical parameter $\gamma$. This means that the nano-resonator frequency rises by increasing the initial gap between the plates and the CNT. Also, figure 3 reveals that improving the applied voltage reduces the frequency of the system. An exciting point is an interaction between the curve and the horizontal axis. For $\eta=50$, the nano-resonator's frequency is zero for geometrical parameters less than 34 .

Similarly, when $\eta=10$, the nano-resonator's frequency is zero for geometrical parameters less than 11 . This is due to the pull-in instability, which is a well-known phenomenon in NEMS/MEMS. In other words, the interactions between the curves and the horizontal axis demonstrate the lowest allowable geometrical parameter for a specific applied voltage. The geometrical parameter at the interaction point can be used for determining the minimum permissible gap, which is an essential parameter for designing the nano-resonators. 


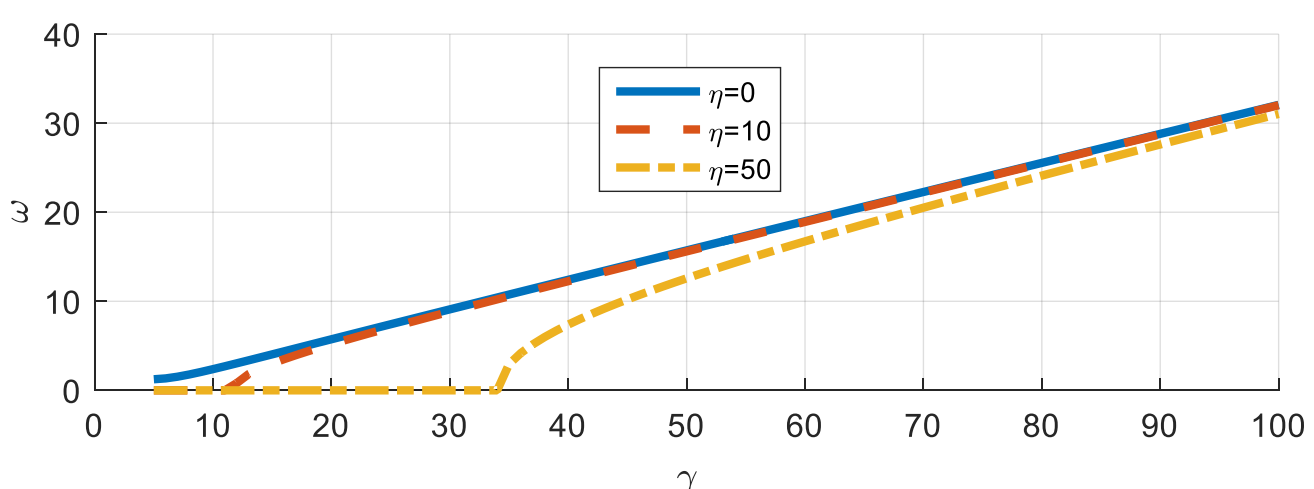

Figure 3. The impact of the geometrical parameter and external voltage on the nano-resonator's frequency

Figure 4 depicts the impact of Casimir force and geometrical parameter on the nano-resonator's frequency. This figure demonstrates that the Casimir force reduces the system frequency. It is clear from Fig. 4 that the impact of Casimir force on the nano-resonators's frequency is more dominant for the lower geometrical parameter. Similar to figure 3, the interaction point with the horizontal axis shows the nano-resonator's pull-in behavior.

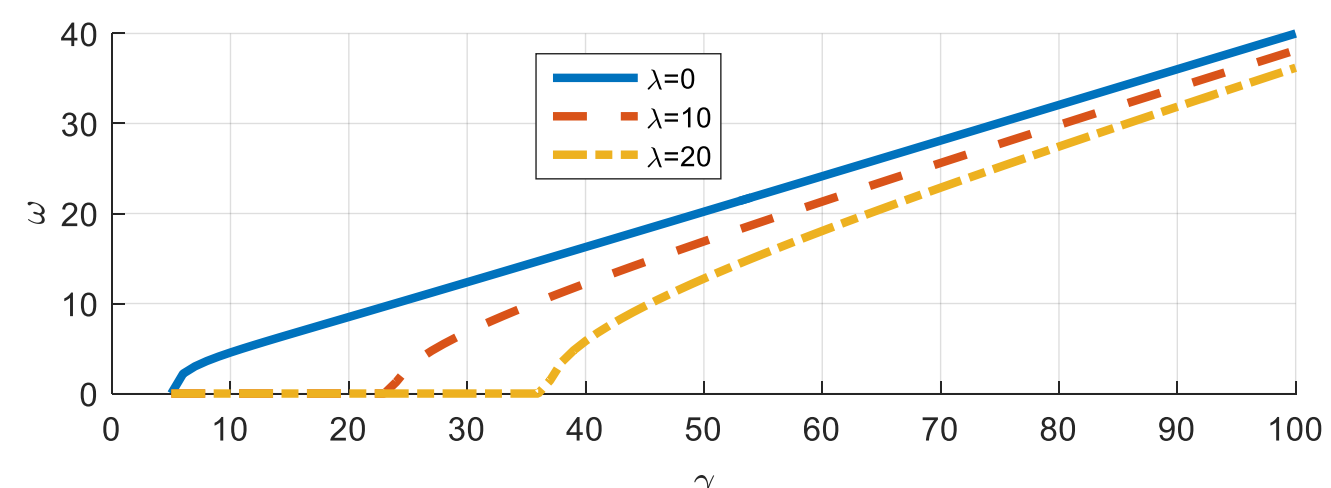

Figure 4. The impact of Casimir force and geometrical parameter on the nano-resonator's frequency

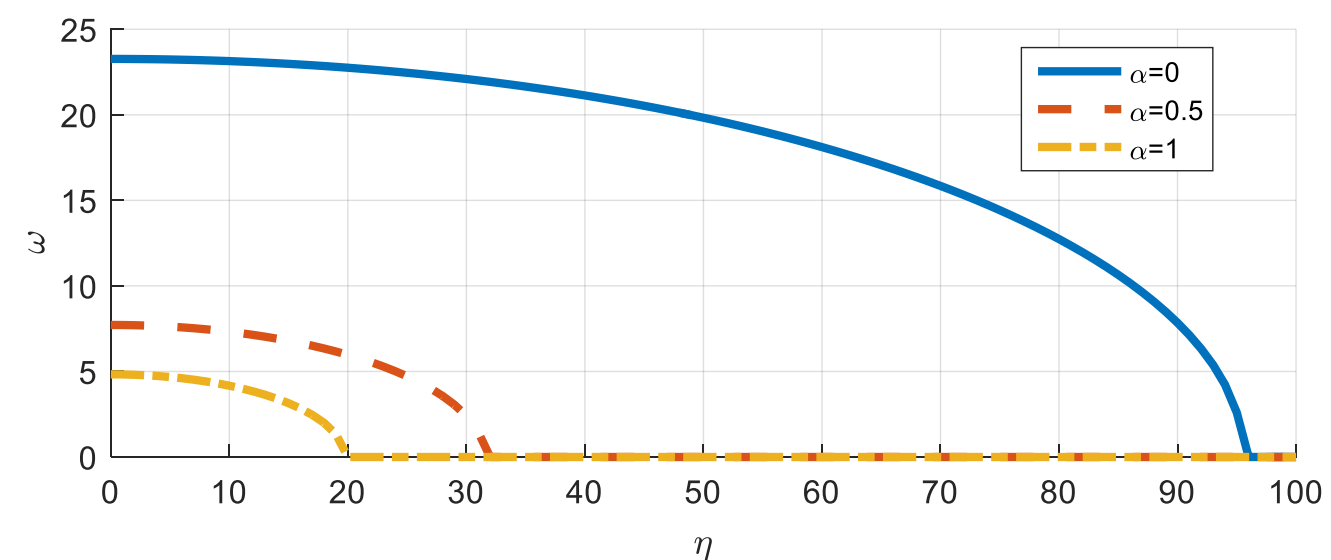

Figure 5. The impact of the nonlocal and geometrical parameter on the nano-resonator's frequency

The frequency of the system for various applied voltage from zero to the pull-in values is demonstrated in Figure 5. The impact of the nonlocal parameter is also shown in this figure. Figure 5 reveals that the nonlocal parameter reduces the system frequency. Figure 5 indicates that the nonlocal parameter enhances the system rigidity and reduces the nano-resonator's pull-in voltage. 


\section{Conclusion}

In this paper, a closed-form solution for investigating the nonlinear oscillation of CNT manufactured is presented. The nano-resonator is considered as a doubly clamped CNT between two fixed plates. The electrical potential is imposed between the CNT and both upper and lower surfaces. The nonlinear governing equation of the nano-resonator is extracted based on the nonlocal elasticity. The impact of the geometrical nonlinearity in the term of mid-plane stretching is incorporated in the constitutive equation. Also, the effect of the Casimir force is taken into account in the developed simulation. The nonlinear partial differential equation of the system transformed into a nonlinear ordinary differential equation. Finally, the energy balance method is employed, and a closed-form solution is obtained for the system's vibrations. The obtained results demonstrated that external electrical force enhances the amplitude of the nano-resonator. However, the nano-resonator's frequency reduces by improving the external electrical potential. Investigating the impact of Casimir force revealed that the influence of Casimir force on the nano-resonator's dynamic performances is similar to electrical force. In other words, the Casimir force enhances the amplitude and reduces the system's frequency. Conversely, the nonlocal parameter has a hardening effect. Improving the nonlocal parameter increases the nano-resonator's frequency and subsequently reduces the system's pull-in voltage.

\section{References}

Ali-Akbari, H., Ceballes, S., \& Abdelkefi, A. (2019). Nonlinear performance analysis of forced carbon nanotube-based bio-mass sensors. International Journal of Mechanics and Materials in Design, 15(2), 291-315.

Amorim, T. D., Dantas, W. G., \& Gusso, A. (2015). Analysis of the chaotic regime of MEMS/NEMS fixedfixed beam resonators using an improved 1DOF model. Nonlinear Dynamics, 79(2), 967-981.

Besley, N. A. (2020). Vibrational Analysis of Carbon Nanotube Based Nanomechanical Resonators. The Journal of Physical Chemistry C.

Dantas, W. G., \& Gusso, A. (2018). Analysis of the chaotic dynamics of MEMS/NEMS doubly clamped beam resonators with two-sided electrodes. International Journal of Bifurcation and Chaos, 28(10), 1850122.

Dequesnes, M., Tang, Z., \& Aluru, N. R. (2004). Static and dynamic analysis of carbon nanotube-based switches. J. Eng. Mater. Technol., 126(3), 230-237.

Eringen, A. C., \& Edelen, D. (1972). On nonlocal elasticity. International Journal of Engineering Science, 10(3), 233-248.

Farrokhabadi, A., Koochi, A., \& Abadyan, M. (2014). Modeling the instability of CNT tweezers using a continuum model. Microsystem Technologies, 20(2), 291-302.

Fu, Y., Zhang, J., \& Wan, L. (2011). Application of the energy balance method to a nonlinear oscillator arising in the microelectromechanical system (MEMS). Current Applied Physics, 11(3), 482-485.

Ganji, S., Ganji, D., \& Karimpour, S. (2009). He's energy balance and He's variational methods for nonlinear oscillations in engineering. International Journal of Modern Physics B, 23(03), 461-471.

Ghalambaz, M., Ghalambaz, M., \& Edalatifar, M. (2015). Buckling Analysis of Cantilever Nanoactuators Immersed in an Electrolyte: A Close Form Solution Using Duan-Rach Modified Adomian Decomposition Method. Journal of Applied and Computational Mechanics, 1(4), 207-219.

Hajjam, A., \& Pourkamali, S. (2011). Fabrication and characterization of MEMS-based resonant organic gas sensors. IEEE Sensors Journal, 12(6), 1958-1964.

Hosseini, S. M. (2018). Analytical solution for nonlocal coupled thermoelasticity analysis in a heat-affected MEMS/NEMS beam resonator based on Green-Naghdi theory. Applied Mathematical Modelling, 57, 21-36.

Jamshidi, N., \& Ganji, D. (2010). Application of energy balance method and variational iteration method to an oscillation of a mass attached to a stretched elastic wire. Current Applied Physics, 10(2), 484-486.

Kang, D.-K., Kim, C.-W., \& Yang, H.-I. (2017). Thermal effects on nonlinear vibration of a carbon nanotubebased mass sensor using finite element analysis. Physica E: Low-dimensional Systems and Nanostructures, 85, 125-136. 
Khosravi, F., Hosseini, S. A., \& Hayati, H. (2020). Free and forced axial vibration of single walled carbon nanotube under linear and harmonic concentrated forces based on nonlocal theory. International Journal of Modern Physics B, 34(08), 2050067.

Kim, P., \& Lieber, C. M. (1999). Nanotube nanotweezers. Science, 286(5447), 2148-2150.

Koochi, A., Farrokhabadi, A., \& Abadyan, M. (2015). Modeling the size dependent instability of NEMS sensor/actuator made of nano-wire with circular cross-section. Microsystem Technologies, 21(2), 355-364.

Liu, H., Li, B., \& Liu, Y. (2019). The inconsistency of nonlocal effect on carbon nanotube conveying fluid and a proposed solution based on local/nonlocal model. European Journal of Mechanics-A/Solids, 78, 103837.

Liu, Y., Song, T., Jia, X., Meng, L., \& Mao, X. (2017). Gold nanoparticles decorated carbon nanotube probe based immunochromatographic assay on cotton thread. Sensors and Actuators B: Chemical, 251, 1112-1118.

Mahmoud, F., Eltaher, M., Alshorbagy, A., \& Meletis, E. (2012). Static analysis of nanobeams including surface effects by nonlocal finite element. Journal of mechanical science and technology, 26(11), 3555-3563.

Mehdipour, I., Ganji, D., \& Mozaffari, M. (2010). Application of the energy balance method to nonlinear vibrating equations. Current Applied Physics, 10(1), 104-112.

Mestrom, R., Fey, R., Van Beek, J., Phan, K., \& Nijmeijer, H. (2008). Modelling the dynamics of a MEMS resonator: simulations and experiments. Sensors and Actuators A: Physical, 142(1), 306-315.

Miandoab, E. M., Yousefi-Koma, A., Pishkenari, H. N., \& Fathi, M. (2014). Nano-resonator frequency response based on strain gradient theory. Journal of Physics D: Applied Physics, 47(36), 365303.

Miandoab, E. M., Yousefi-Koma, A., Pishkenari, H. N., \& Tajaddodianfar, F. (2015). Study of nonlinear dynamics and chaos in MEMS/NEMS resonators. Communications in Nonlinear Science and Numerical Simulation, 22(1-3), 611-622.

Ouakad, H. M., \& Younis, M. I. (2010). Nonlinear dynamics of electrically actuated carbon nanotube resonators. Journal of computational and nonlinear dynamics, 5(1).

Pashaki, P. V., \& Ji, J.-C. (2020). Nonlocal nonlinear vibration of an embedded carbon nanotube conveying viscous fluid by introducing a modified variational iteration method. Journal of the Brazilian Society of Mechanical Sciences and Engineering, 42(4), 1-13.

Qian, Z., Hui, Y., Liu, F., Kang, S., Kar, S., \& Rinaldi, M. (2016). Graphene-aluminum nitride NEMS resonant infrared detector. Microsystems \& nanoengineering, 2(1), 1-7.

Rahmani, O., Shokrnia, M., Golmohammadi, H., \& Hosseini, S. (2018). Dynamic response of a single-walled carbon nanotube under a moving harmonic load by considering modified nonlocal elasticity theory. The European Physical Journal Plus, 133(2), 1-13.

Sedighi, H. M., \& Farjam, N. (2017). A modified model for dynamic instability of CNT based actuators by considering rippling deformation, tip-charge concentration and Casimir attraction. Microsystem Technologies, 23(6), 2175-2191.

Tajaddodianfar, F., Yazdi, M. R. H., \& Pishkenari, H. N. (2017). Nonlinear dynamics of MEMS/NEMS resonators: analytical solution by the homotopy analysis method. Microsystem Technologies, 23(6), 19131926.

Tocchio, A., Caspani, A., \& Langfelder, G. (2011). Mechanical and electronic amplitude-limiting techniques in a MEMS resonant accelerometer. IEEE Sensors Journal, 12(6), 1719-1725.

Yang, Y., Wang, J., \& Yu, Y. (2018). Wave propagation in fluid-filled single-walled carbon nanotube based on the nonlocal strain gradient theory. Acta Mechanica Solida Sinica, 31(4), 484-492.

Zeighampour, H., Beni, Y. T., \& Karimipour, I. (2017). Wave propagation in double-walled carbon nanotube conveying fluid considering slip boundary condition and shell model based on nonlocal strain gradient theory. Microfluidics and Nanofluidics, 21(5), 85. 